\title{
MEDUSAE OF THE GENERA PARAPHYLLINA, PERIPHYLLA AND ATOLLA FROM THE AMSTERDAM MID NORTH ATLANTIC PLANKTON EXPEDITIONS (1980-1983)*
}

\author{
by \\ S. VAN DER SPOEL \\ Institute of Taxonomic Zoology, University of Amsterdam, P.O. Box 20125, 1000 HC Amsterdam, \\ The Netherlands
}

\begin{abstract}
The vertical distribution patterns of Paraphyllina ransoni Russell, 1956, Periphylla periphylla (Péron Lesueur, 1809), Atolla parva Russell, 1958, A. vanhoeffeni Russell, 1957, and $A$. wyvillei Haeckel, 1880 are described. A comparison with other bathypelagic taxa shows that there are various upper limits to be drawn for the bathypelagic area. Four typically different vertical distribution patterns are described.

\section{RÉSUMÉ}

Les types de distribution verticale de Paraphyllina ransoni Russell, 1956, Periphylla periphylla (Péron \& Lesueur, 1809), Atolla parva Russell, 1958, A. vanhoeffeni Russell, 1957 , et $A$. wyvillei Haeckel, 1880 sont décrits. Une comparaison avec d'autres taxa bathypélagiques montre que les limites supérieures dans le cadre du domaine bathypélagique sont différentes. Quatre types distincts de distribution verticale sont décrits.
\end{abstract}

\section{INTRODUCTION}

To study the vertical subdivision of the water column, especially with regard to the upper levels of the bathypelagic realm, nemerteans (Van der Spoel, 1985a) and coronate medusae have been studied. For the nemerteans it became clear that Nectonemertes mirabilis Verrill, 1892 shows different vertical distribution patterns in the different seasons sampled. From the other nemerteans it could be concluded that the upper limit of this bathypelagic group is found between depths of $500 \mathrm{~m}$ and $1000 \mathrm{~m}$. For the

- AMNAPE Project 101A. Report no. 28, supported by the Netherlands Ministry of Education and Sciences. (Report no. 27 appeared in Biol. Oceanogr., 4 (2): 439-445-1987.) bathypelagic coronate medusae rather shallow and sometimes restricted occurrences are known. It seems worth while to compare the vertical distributions of the nemerteans and coronates with the bathymetric distribution of the chaetognath Sagitta planctonis Steinhaus, 1896 and the fish Argyropelecus spp.

The term bathypelagic is ill-defined, and used in different ways as is shown by the citations below:

"The term epipelagic denotes the upper water layers... In the present work it will only comprise the upper $150-200 \mathrm{~m}$... Below this epipelagic layer lies the deepwater with its bathypelagic fauna"' (Ekman, 1953: 312).

"If... the transition between this zone" [the mesopelagic] "and the dark underlying bathypelagic zone is the threshold of light...". "The bathypelatic zone is thus uniformly cold and dark..." (Marshall, 1979: 43, 44).

"Chun $(1887,1888)$ introduced the term 'interzonal species' for animals inhabiting both deep-sea and surface zones. More widely used is the expression 'bathypelagic species' proposed by Haeckel (1891), for designating the migrations of animals found at different depths at different times. Among the Russian authors, Brodskii (1950, 1952), Vinogradov (1954a, $1955 \mathrm{a}, \mathrm{c}$ ) and others apply this term as defined by Haeckel. On the other hand Hjort (see Murray and Hjort, 1912) first used the name 'bathypelagic' in a wider sense to cover all the pelagic animals of the deep-seas"' (Vinogradov, 1970: 43).

"The mesopelagic zone is subdivided into shallow mesopelagic $(300-700 \mathrm{~m})$ and deep mesopelagic (700-1000 m)"' (Boxshall, 1981: 
153). This author considers the bathypelagic to start below $1000 \mathrm{~m}$.

Some authors consider the bathy- (and meso-) pelagial to be physicochemically determined water masses, others state that the fauna determines the realm, but most authors use the terms bathy- and mesopelagic for animals as well as for water layers, while sometimes these terms are even used to indicate the behaviour of the animals. This is most confusing; in this paper it is tried to detect, for animals commonly considered to be "bathypelagic" in behaviour and distribution, the upper limit of their vertical range.

\section{MATERIAL}

The material studied was collected by the Amsterdam Mid North Atlantic Plankton Expeditions held during four years (1980-1983) in different seasons, between $55^{\circ} \mathrm{N}$ and $24^{\circ} \mathrm{N}$ along approximately the $30^{\circ} \mathrm{W}$ meridian, in depth strata of usually $100 \mathrm{~m}$ (500 m below $500 \mathrm{~m}$ depth) thickness (Van der Spoel, 1981, 1985b; Van der Spoel \& Meerding, 1983). The majority of the samples was preserved in $4 \%$ formalin and after approximately one year transferred to propylene-phenoxetol/propylene-glycol (Heyman, 1981). All specimens were identified and measured. The numbers used in this paper for abundances are the actual numbers collected without correction for fishing effort or net type used. As the abundance comparisons are roughly made this method seems to be acceptable.

TABLE I

Records of Paraphyllina ransoni (only collected in summer 1983; Tr. = trawl).

\begin{tabular}{|c|c|c|c|c|c|c|}
\hline \multirow[t]{2}{*}{ Sta. } & \multirow[t]{2}{*}{ Tr. } & \multirow[t]{2}{*}{$N$} & \multicolumn{2}{|c|}{ Position } & \multirow{2}{*}{$\begin{array}{r}\text { Depth } \\
\text { (m) }\end{array}$} & \multirow[t]{2}{*}{ Time } \\
\hline & & & North & West & & \\
\hline 74 & 10 & 2 & $54^{\circ} 20.9^{\prime} \mathrm{N}$ & $29^{\circ} 53.7^{\prime} \mathrm{W}$ & $1000-1750$ & at night \\
\hline 75 & 1 & 11 & $52^{\circ} 56.5^{\prime} \mathrm{N}$ & $29^{\circ} 53.8^{\prime} \mathrm{W}$ & $200-300$ & by day \\
\hline 75 & 6 & 34 & $53^{\circ} 01.0^{\prime} \mathrm{N}$ & $29^{\circ} 51.3^{\prime} \mathrm{W}$ & $90-200$ & by day \\
\hline 78 & 55 & 1 & $44^{\circ} 58.3^{\prime} \mathrm{N}$ & $29^{\circ} 52.4^{\prime} \mathrm{W}$ & $302-400$ & at dawn \\
\hline 78 & 57 & 7 & $45^{\circ} 00.6^{\prime} \mathrm{N}$ & $29^{\circ} 55.9^{\prime} \mathrm{W}$ & $398-500$ & at dawn \\
\hline 81 & 11 & 7 & $41^{\circ} 03.0^{\prime} \mathrm{N}$ & $35^{\circ} 29.3^{\prime} \mathrm{W}$ & $200-300$ & at night \\
\hline 84 & 5 & 2 & $35^{\circ} 08.6^{\prime} \mathrm{N}$ & $31^{\circ} 23.8^{\prime} \mathrm{W}$ & $100-150$ & at night \\
\hline
\end{tabular}

\section{RESULTS}

Paraphyllina ransoni Russell, 1956 (Fig. 1)

This rather rare species was hitherto only reported from $48^{\circ} 26^{\prime} \mathrm{N} 09^{\circ} 42^{\prime} \mathrm{W}, 900 \mathrm{~m}$ depth (45 specimens), $\quad 04^{\circ} 58^{\prime} \mathrm{N} \quad 04^{\circ} 02^{\prime} \mathrm{W} \quad(610$ specimens), the shore of Villefranche (1 specimen) and off the West coast of Africa (1 specimen) (Russell, 1970). We found it only during the summer cruise in 1983. The vertical distribution pattern closely resembles that of Periphylla periphylla (figs. 2-4), though scarcity of material gives an incomplete picture (cf. table I).

This species occurs over a considerable depth range from 90 to $1750 \mathrm{~m}$ and probably more, while its north-south range known so far is from $54^{\circ} \mathrm{N}$ to $04^{\circ} \mathrm{N}$ in the Atlantic Ocean. It is striking that this species has only been found in summer 1983.

Periphylla periphylla (Péron \& Lesueur, 1809) (Figs. 2-4)

This species, usually considered bathypelagic, can better be described as mesopelagic since its main distribution is found between 200 and $1200 \mathrm{~m}$ (see table II). In the deeper trawls from 1000 to $1750 \mathrm{~m}$, made during the summer cruise of 1983 , it was absent south of $50^{\circ} \mathrm{N}$. The occurrence at shallow depths directly below 50 $\mathrm{m}$ is mainly restricted to the night periods in 1980-1982, though during the summer (1983) cruise specimens were also collected above 100 $\mathrm{m}$ by day. The vertical range is situated in the North Atlantic mixed waters (cf. Van der Spoel 
\& Heyman, 1983). A temperature-dependent distribution is not found, but none of the other coronates showed this either.

Between $46^{\circ}$ and $42^{\circ} \mathrm{N}$ the population of $P$. periphylla changes strongly. North of this belt the diameter of the bell varies from 3-235 mm while it ranges from $3-45 \mathrm{~mm}$ in the area south of $42^{\circ} \mathrm{N}$. Moreover, the samples south of $42^{\circ} \mathrm{N}$ only provided less than 10 specimens per station, while in the northern area all stations yielded more than 30 specimens. The larger numbers and specimens of greater size north of $42^{\circ} \mathrm{N}$, where the species lives also at shallow depths, were not all collected shallower as might have been expected from comparison of the northern and southern area. Still, it cannot be accepted that the deeper and the more southward living specimens are merely expatriated specimens, as smaller specimens south of $42^{\circ} \mathrm{N}$ all show more developed gonads than do specimens of the same size collected in the north. Moreover, expatriated specimens do usually grow larger than local ones. Kramp (1913) also found, in Greenland waters, specimens of every size at every depth and smaller specimens more abundant in the deeper layers. Stiasny (1934) did not agree with Kramp's findings but his data may have been biased as his material came from different water masses some of which affected by upwelling. Though Mauchline \& Harvey (1983) found indications for diurnal vertical migration in both $A$. wyvillei and $P$. periphylla, the present material provided only slight indications for such a phenomenon in the latter species.

The number of specimens collected in each trawl differs strongly in the four seasons, viz.: from an average of 4.7 in winter to 15.3 in autumn.

Atolla parva Russell, 1958

(Figs. 5-6)

In this species specimens with 20 and 24 tentacles at the bell margin were treated separately, as Russell $(1959,1970)$ mentioned that probably a taxonomic variation is concerned here. The 20-tentacle form seems to have a slightly smaller north-south range than the 24-tentacle form as is shown in figs. 5 and 6. Rarely specimens with 21 (1 spec. Sta. 78-1), 22 (1 spec. Sta. 50-2, 1 spec. Sta. 51-12) and 23 tentacles (1 spec. Sta. 45-11, 1 spec. Sta. 78-39 and 1 spec. Sta. 84-24) were found.

The areas with higher abundance of both types point to a difference; the 20-tentacle form dominates south of $42^{\circ} \mathrm{N}$ while the other form usually dominates north of $40^{\circ} \mathrm{N}$. This is also in agreement with the opinion of Russell (1970) that tentacle numbers are higher (up to 26) in the north. The depth range of both types is 500$1750 \mathrm{~m}$ with exceptional occurrences up to 400 $\mathrm{m}$ in autumn and spring. Unlike the preceding species, this species shows no shallower occurrence in colder waters. The number of specimens collected per trawl only slightly fluctuates in the different seasons around an average of 4.7 specimens per trawl, with a minimum of 3.3. The variation in size mentioned by Casanova (1977) is not affirmed in the present material; however, the open ocean waters represent other conditions which may explain this difference (cf. table II).

\section{Atolla vanhoeffeni Russell, 1957 (Fig. 7)}

This species of the central North Atlantic waters (Russell, 1970) shows its highest abundance between $40^{\circ}$ and $45^{\circ} \mathrm{N}$. Its vertical range does neither become shallower in the northern part of its range, nor does it seem to be temperature-dependent. The bathymetric range is 400 (300 in spring) $-1000 \mathrm{~m}$. In all samples taken from depths below $1000 \mathrm{~m}$ (summer cruise 1983) this species is completely absent, while the other species discussed do occur in these samples. As a consequence this species can be considered deep-mesopelagic, restricted to depths between 300 and $1000 \mathrm{~m}$. Casanova (1981) also found for this species a restricted vertical range between 300 and $400 \mathrm{~m}$ in the tropics, while Mauchline \& Harvey (1983) found a range of $220-500 \mathrm{~m}$ in the Rockall Trough. In the present collections relatively high numbers of this species were 


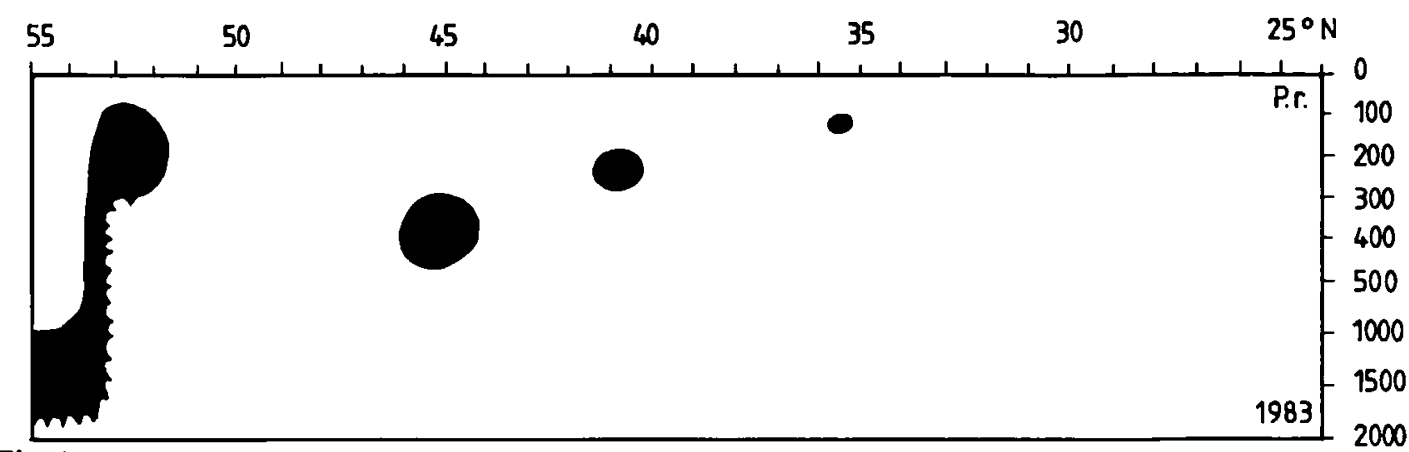

Fig.1

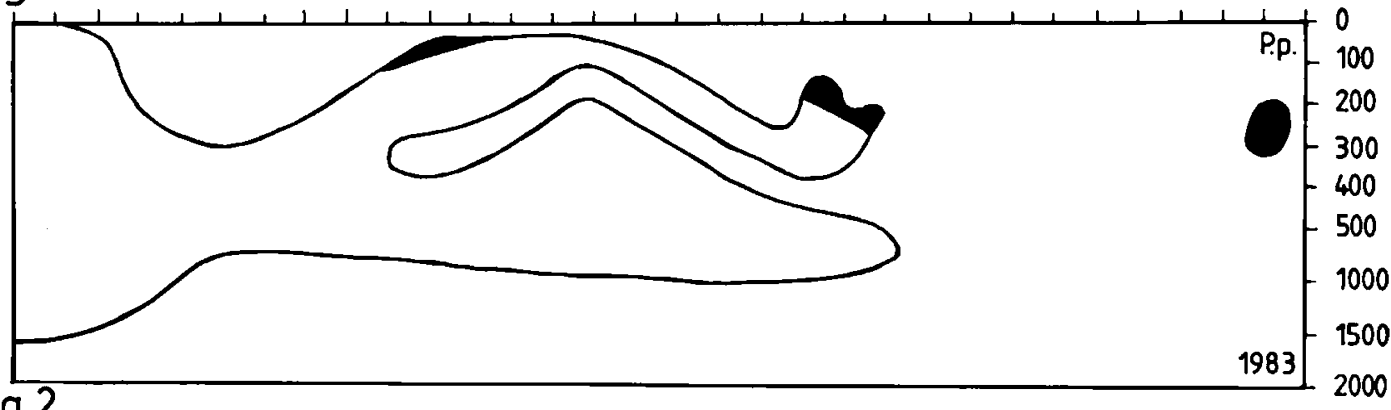

Fig. 2 2000

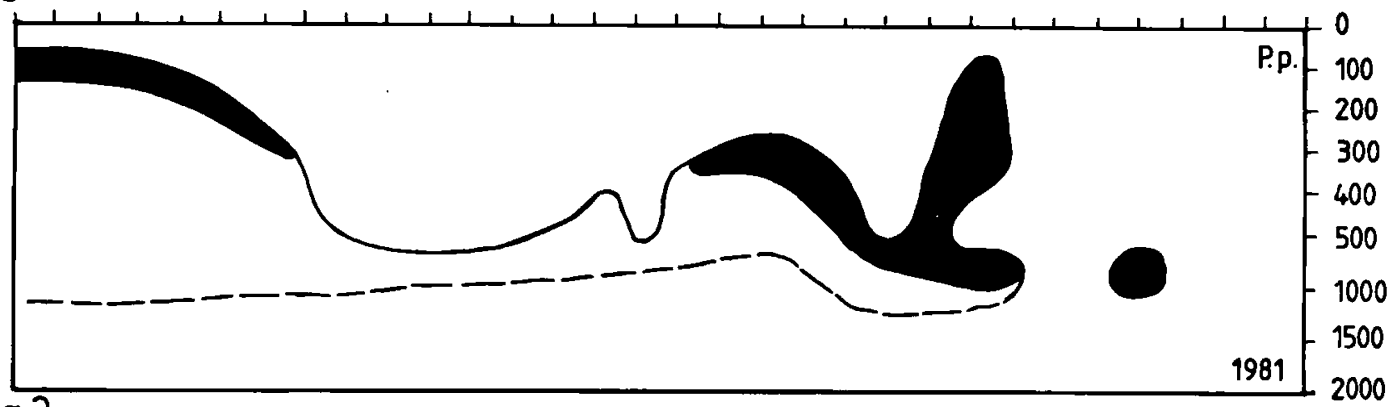

Fig.3

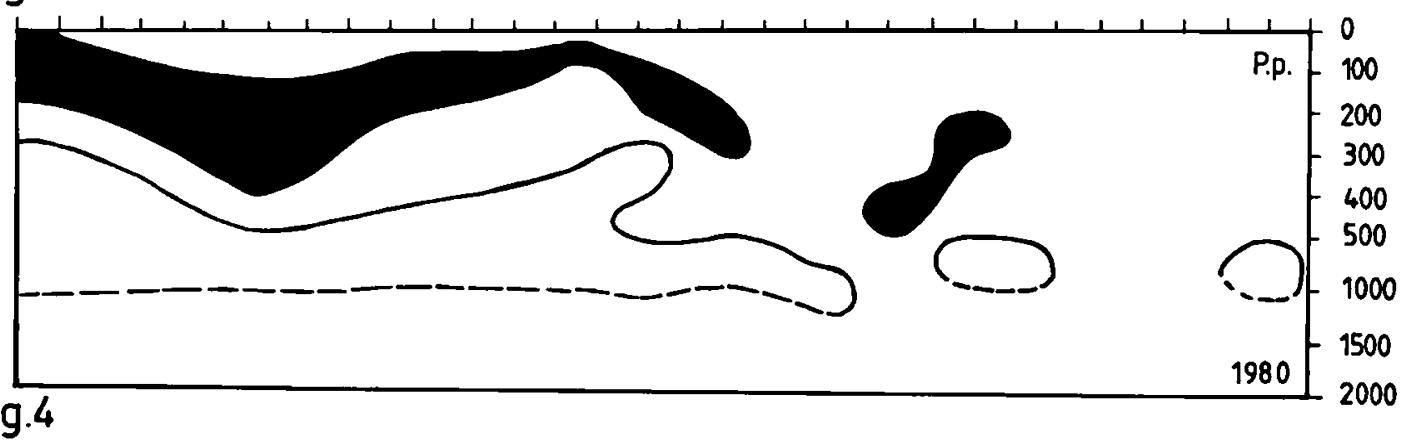

Fig. 1. Vertical distribution of Paraphyllina ransoni in summer 1983 along the cruise transect between $55^{\circ} \mathrm{N}$ and $24^{\circ} \mathrm{N}$ Depth (m) along the vertical axis.

Figs. 2-4. Vertical distribution of Periphylla periphylla along the cruise transect between $55^{\circ} \mathrm{N}$ and $24^{\circ} \mathrm{N}$ for summer 1983 (fig. 2), autumn 1981 (fig. 3) and spring 1980 (fig. 4). Depth (m) along the vertical axis. Areas with exclusively night samples are black. 
TABLE II

Numbers of specimens of Periphylla periphylla (P.p.), Atolla vanhoeffeni (A.v.), A. wyvillei (A.w.) and A. parva (A.p.) by station number and trawl. After the number of specimens the average bell diameter $(\mathrm{mm})$ per sample is given. In $A$. parva specimens with 20 and 24 tentacles are treated separately.

\begin{tabular}{|c|c|c|c|c|c|c|c|c|c|}
\hline \multirow[t]{2}{*}{ Sta. } & \multirow{2}{*}{ Tr. } & \multirow{2}{*}{ P.p. } & \multirow[t]{2}{*}{ A.v. } & \multirow[t]{2}{*}{ A.w. } & \multirow{2}{*}{$\begin{array}{l}\text { A.p. } \\
20 \mathrm{t}\end{array}$} & \multirow{2}{*}{$\begin{array}{l}A . p . \\
24 \mathrm{t}\end{array}$} & \multicolumn{2}{|c|}{ Position } & \multirow{2}{*}{$\begin{array}{c}\text { Depth } \\
\text { (m) }\end{array}$} \\
\hline & & & & & & & North & West & \\
\hline \multicolumn{10}{|c|}{ Spring 1980} \\
\hline 10 & 1 & $2-20$ & & & & & $54^{\circ} 57.4^{\prime}$ & $30^{\circ} 00.0^{\prime}$ & $390-510$ \\
\hline 10 & 3 & 55-? & & & & & $54^{\circ} 54.0^{\prime}$ & $30^{\circ} 32.2^{\prime}$ & $480-1010$ \\
\hline 10 & 5 & $11-152$ & & & & & $54^{\circ} 44.9^{\prime}$ & $29^{\circ} 56.4^{\prime}$ & $265-400$ \\
\hline 10 & 7 & $14-14$ & & & & & $54^{\circ} 43.1^{\prime}$ & $29^{\circ} 58.9^{\prime}$ & $0-170$ \\
\hline 11 & 1 & $89-10$ & $1-14$ & $4-31$ & & $4-29$ & $53^{\circ} 00.8^{\prime}$ & $29^{\circ} 58.1^{\prime}$ & $290-995$ \\
\hline 11 & 2 & $2-145$ & & & & & $53^{\circ} 01.3^{\prime}$ & $29^{\circ} 51.1^{\prime}$ & $92-210$ \\
\hline 11 & 4 & $3-42$ & & & & & $53^{\circ} 08.1^{\prime}$ & $29^{\circ} 53.9^{\prime}$ & $40-100$ \\
\hline 11 & 7 & $9-130$ & & & & & $53^{\circ} 08.2^{\prime}$ & $29^{\circ} 55.5^{\prime}$ & $0-50$ \\
\hline 13 & 2 & $6-14$ & & & & & $49^{\circ} 01.1^{\prime}$ & $30^{\circ} 00.6^{\prime}$ & $130-220$ \\
\hline 13 & 3 & $37-<20$ & & & & & $49^{\circ} 00.9^{\prime}$ & $29^{\circ} 42.7^{\prime}$ & $215-310$ \\
\hline 13 & 4 & $93-13$ & $5-9$ & & & & $48^{\circ} 59.8^{\prime}$ & $29^{\circ} 35.1^{\prime}$ & $310-400$ \\
\hline 13 & 6 & $1-43$ & $1-7$ & & & & $49^{\circ} 00.7^{\prime}$ & $29^{\circ} 31.0^{\prime}$ & $375-500$ \\
\hline 13 & 9 & $31-15$ & $9-17$ & $9-33$ & & $3-13$ & $49^{\circ} 00.8^{\prime}$ & $29^{\circ} 18.5^{\prime}$ & $480-1005$ \\
\hline 14 & 5 & $15-?$ & $7-8$ & $8-39$ & $2-7$ & $4-11$ & $45^{\circ} 15.0^{\prime}$ & $29^{\circ} 50.0^{\prime}$ & $430-1000$ \\
\hline 14 & 8 & 26-12 & & & & & $45^{\circ} 21.8^{\prime}$ & $29^{\circ} 46.4^{\prime}$ & $85-200$ \\
\hline 14 & 10 & $1-11$ & & & & & $45^{\circ} 24.1^{\prime}$ & $29^{\circ} 40.6^{\prime}$ & $50-100$ \\
\hline 16 & 3 & $2-19$ & $2-7$ & 4-39 & $12-9$ & $3-16$ & $41^{\circ} 47.8^{\prime}$ & $35^{\circ} 02.8^{\prime}$ & $490-1000$ \\
\hline 17 & 1 & $3-16$ & & & & & $41^{\circ} 01.4^{\prime}$ & $35^{\circ} 31.3^{\prime}$ & 45-95 \\
\hline 17 & 2 & $1-20$ & $24-10$ & & & & $41^{\circ} 10.6^{\prime}$ & $35^{\circ} 30.9^{\prime}$ & $330-505$ \\
\hline 18 & 1 & $1-38$ & $3-13$ & $6-30$ & $6-9$ & & $39^{\circ} 58.5^{\prime}$ & $36^{\circ} 24.9^{\prime}$ & $520-1130$ \\
\hline 18 & 2 & $1-17$ & $21-3$ & & & & $39^{\circ} 52.2^{\prime}$ & $36^{\circ} 18.1^{\prime}$ & $265-430$ \\
\hline 18 & 4 & $1-25$ & & & & & $39^{\circ} 47.2^{\prime}$ & $36^{\circ} 16.1^{\prime}$ & $110-205$ \\
\hline 18 & 10 & $2-11$ & $10-9$ & & & & $39^{\circ} 53.9^{\prime}$ & $35^{\circ} 38.9^{\prime}$ & $440-910$ \\
\hline 19 & 1 & 4-14 & & & & & $38^{\circ} 00.6^{\prime}$ & $35^{\circ} 29.7^{\prime}$ & $190-320$ \\
\hline 19 & 22 & $1-10$ & $10-10$ & $1-24$ & $9-9$ & $3-7$ & $37^{\circ} 48.5^{\prime}$ & $35^{\circ} 17.4^{\prime}$ & $500-1000$ \\
\hline 20 & 1 & & $9-9$ & & 1-5 & & $35^{\circ} 27.2^{\prime}$ & $31^{\circ} 51.6^{\prime}$ & $505-870$ \\
\hline 20 & 3 & $1-14$ & 5-11 & $13-23$ & $25-11$ & $8-15$ & $35^{\circ} 22.7^{\prime}$ & $31^{\circ} 44.9^{\prime}$ & $770-1250$ \\
\hline 21 & 3 & $1-20$ & $3-10$ & & & & $33^{\circ} 47.3^{\prime}$ & $30^{\circ} 41.8^{\prime}$ & $390-530$ \\
\hline 21 & 6 & & $8-10$ & $2-42$ & $7-9$ & $2-18$ & $33^{\circ} 40.5^{\prime}$ & $30^{\circ} 40.6^{\prime}$ & $510-1000$ \\
\hline 22 & 1 & $1-20$ & $5-6$ & & $1-9$ & $1-11$ & $32^{\circ} 19.0^{\prime}$ & $30^{\circ} 03.1^{\prime}$ & $500-1000$ \\
\hline 22 & 6 & $.3-8$ & & & & & $32^{\circ} 04.1^{\prime}$ & $29^{\circ} 54.0^{\prime}$ & $195-300$ \\
\hline 23 & 2 & $1-11$ & $1-6$ & $1-4$ & $3-9$ & $4-14$ & $30^{\circ} 39.9^{\prime}$ & $29^{\circ} 59.5^{\prime}$ & $505-960$ \\
\hline 25 & 1 & & $1-30$ & & & $1-18$ & $28^{\circ} 42.0^{\prime}$ & $29^{\circ} 59.1^{\prime}$ & $490-1000$ \\
\hline 26 & 4 & $1-27$ & & & & $1-11$ & $24^{\circ} 52.0^{\prime}$ & $29^{\circ} 59.5^{\prime}$ & $510-1090$ \\
\hline 27 & 10 & & $1-8$ & & & $1-15$ & $24^{\circ} 48.6^{\prime}$ & $28^{\circ} 47.2^{\prime}$ & $475-1000$ \\
\hline \multicolumn{10}{|c|}{ Summer 1983} \\
\hline 74 & 1 & $2-90$ & & & & & $54^{\circ} 20.8^{\prime}$ & $29^{\circ} 57.1^{\prime}$ & $296-400$ \\
\hline 74 & 4 & $1-235$ & & & & & $54^{\circ} 24.7^{\prime}$ & $30^{\circ} 00.4^{\prime}$ & $200-300$ \\
\hline 74 & 6 & $2-130$ & & & & & $54^{\circ} 27.1^{\prime}$ & $30^{\circ} 01.3^{\prime}$ & $100-200$ \\
\hline 74 & 7 & $9-103$ & & & & & $54^{\circ} 23.3^{\prime}$ & $29^{\circ} 58.3^{\prime}$ & 5-52 \\
\hline 74 & 10 & $2-110$ & & $4-57$ & $1-7$ & $2-25$ & $54^{\circ} 20.9^{\prime}$ & $29^{\circ} 53.7^{\prime}$ & $1000-1750$ \\
\hline 74 & 11 & $2-90$ & & & & & $54^{\circ} 18.0^{\prime}$ & $29^{\circ} 49.6^{\prime}$ & $50-98$ \\
\hline 74 & 12 & $7-14$ & $1-5$ & & & & $54^{\circ} 22.0^{\prime}$ & $29^{\circ} 49.0^{\prime}$ & $400-502$ \\
\hline 74 & 15 & $33-14$ & $1-7$ & $2-71$ & $2-24$ & & $54^{\circ} 24.4^{\prime}$ & $29^{\circ} 48.0^{\prime}$ & $490-995$ \\
\hline 75 & 1 & $18-33$ & & & & & $52^{\circ} 56.5^{\prime}$ & $29^{\circ} 53.8^{\prime}$ & $200-300$ \\
\hline 75 & 6 & $12-31$ & & & & & $53^{\circ} 01.0^{\prime}$ & $29^{\circ} 51.3^{\prime}$ & $90-200$ \\
\hline 76 & 26 & $7-22$ & $1-2$ & $20-28$ & $1-39$ & $5-13$ & $50^{\circ} 21.3^{\prime}$ & $29^{\circ} 29.7^{\prime}$ & $500-995$ \\
\hline 76 & 30 & $1-154$ & & & & & $50^{\circ} 20.0^{\prime}$ & $29^{\circ} 20.7^{\prime}$ & $400-500$ \\
\hline
\end{tabular}


TABLE II (continuation)

\begin{tabular}{|c|c|c|c|c|c|c|c|c|c|}
\hline \multirow[t]{2}{*}{ Sta. } & \multirow[t]{2}{*}{ Tr. } & \multirow[t]{2}{*}{ P.p. } & \multirow[t]{2}{*}{ A.v. } & \multirow[t]{2}{*}{ A.w. } & \multirow{2}{*}{$\begin{array}{l}\text { A.p. } \\
20 \mathrm{t}\end{array}$} & \multirow{2}{*}{$\begin{array}{l}A . p . \\
24 \mathrm{t}\end{array}$} & \multicolumn{2}{|c|}{ Position } & \multirow{2}{*}{$\begin{array}{c}\text { Depth } \\
\text { (m) }\end{array}$} \\
\hline & & & & & & & North & West & \\
\hline $\begin{array}{l}76 \\
78\end{array}$ & $\begin{array}{r}31 \\
1\end{array}$ & $1-?$ & $7-9$ & & $1-54$ & $1-10$ & $\begin{array}{l}50^{\circ} 22.0^{\prime} \\
44^{\circ} 58.5^{\prime}\end{array}$ & $\begin{array}{l}29^{\circ} 23.7^{\prime} \\
30^{\circ} 03.8^{\prime}\end{array}$ & $\begin{array}{l}300-398 \\
500-1000\end{array}$ \\
\hline 78 & 39 & $3-32$ & $3-9$ & $6-32$ & & $2-18$ & $45^{\circ} 02.3^{\prime}$ & $30^{\circ} 01.3^{\prime}$ & $500-1000$ \\
\hline 78 & 49 & $1-20$ & & & & & $44^{\circ} 59.6^{\prime}$ & $30^{\circ} 06.9^{\prime}$ & $196-299$ \\
\hline 78 & 53 & $7-16$ & & & & & $44^{\circ} 57.0^{\prime}$ & $30^{\circ} 01.0^{\prime}$ & $50-98$ \\
\hline 78 & 54 & $1-35$ & & & & & $44^{\circ} 56.8^{\prime}$ & $29^{\circ} 56.1^{\prime}$ & $98-200$ \\
\hline 78 & 55 & $2-6$ & $12-9$ & & & & $44^{\circ} 58.3^{\prime}$ & $29^{\circ} 52.4^{\prime}$ & $302-400$ \\
\hline 78 & 57 & & $12-7$ & & & & $44^{\circ} 58.1^{\prime}$ & $29^{\circ} 52.1^{\prime}$ & $0-50$ \\
\hline 78 & 60 & & & $5-53$ & $5-6$ & $2-35$ & $45^{\circ} 33.1^{\prime}$ & $29^{\circ} 59.1^{\prime}$ & $1002-1752$ \\
\hline 81 & 6 & & $10-13$ & $7-24$ & & $1-21$ & $40^{\circ} 56.2^{\prime}$ & $35^{\circ} 31.6^{\prime}$ & $500-1000$ \\
\hline 81 & 8 & $1-24$ & & & & & $41^{\circ} 02.3^{\prime}$ & $35^{\circ} 32.3^{\prime}$ & $35-100$ \\
\hline 81 & 11 & $7-14$ & & & & & $41^{\circ} 03.0^{\prime}$ & $35^{\circ} 29.3^{\prime}$ & $200-300$ \\
\hline 81 & 14 & $2-19$ & $10-9$ & & & & $40^{\circ} 59.1^{\prime}$ & $35^{\circ} 25.4^{\prime}$ & $280-400$ \\
\hline 81 & 16 & $2-17$ & $29-8$ & & & & $40^{\circ} 56.6^{\prime}$ & $35^{\circ} 29.6^{\prime}$ & $400-500$ \\
\hline 81 & 17 & $5-29$ & $3-16$ & $8-18$ & $1-9$ & $1-12$ & $40^{\circ} 58.5^{\prime}$ & $35^{\circ} 27.5^{\prime}$ & $505-1000$ \\
\hline 84 & 24 & $1-16$ & $3-13$ & $2-33$ & $3-8$ & $2-11$ & $35^{\circ} 09.6^{\prime}$ & $31^{\circ} 31.8^{\prime}$ & $494-1000$ \\
\hline 84 & 35 & $1-40$ & & & & & $35^{\circ} 09.2^{\prime}$ & $31^{\circ} 29.3^{\prime}$ & $205-305$ \\
\hline 84 & 36 & $1-44$ & & & & & $35^{\circ} 11.0^{\prime}$ & $31^{\circ} 30.9^{\prime}$ & $300-400$ \\
\hline 84 & 37 & $2-44$ & $5-13$ & $5-29$ & $4-9$ & $1-19$ & $35^{\circ} 11.8^{\prime}$ & $31^{\circ} 31.4^{\prime}$ & $505-1000$ \\
\hline 84 & 71 & $1-14$ & & & & & $35^{\circ} 09.9^{\prime}$ & $31^{\circ} 30.6^{\prime}$ & $200-255$ \\
\hline 84 & 72 & & $2-6$ & & & & $35^{\circ} 09.2^{\prime}$ & $31^{\circ} 26.6^{\prime}$ & $405-495$ \\
\hline 84 & 76 & $1-10$ & & & & & $35^{\circ} 09.3^{\prime}$ & $31^{\circ} 27.4^{\prime}$ & $145-205$ \\
\hline 84 & 77 & & & $7-14$ & $7-9$ & & $35^{\circ} 09.6^{\prime}$ & $31^{\circ} 31.7^{\prime}$ & $1000-1750$ \\
\hline 87 & 35 & & & & $2-9$ & $1-15$ & $30^{\circ} 00.9^{\prime}$ & $29^{\circ} 04.0^{\prime}$ & $500-1000$ \\
\hline 987 & 29 & & & & & $2-12$ & $29^{\circ} 59.8^{\prime}$ & $27^{\circ} 45.7^{\prime}$ & $752-1005$ \\
\hline 89 & 8 & & $2-16$ & & & $2-9$ & $24^{\circ} 49.5^{\prime}$ & $30^{\circ} 01.6^{\prime}$ & $510-1000$ \\
\hline 89 & 29 & & $3-14$ & & & & $24^{\circ} 52.8^{\prime}$ & $30^{\circ} 03.5^{\prime}$ & $515-1000$ \\
\hline 89 & 34 & $2-35$ & & & & & $24^{\circ} 54.3^{\prime}$ & $30^{\circ} 00.6^{\prime}$ & $300-400$ \\
\hline 89 & 35 & $2-45$ & & & & & $24^{\circ} 49.7^{\prime}$ & $30^{\circ} 01.7^{\prime}$ & $190-300$ \\
\hline \multicolumn{10}{|c|}{ Autumn 1981} \\
\hline 36 & 8 & $3-36$ & & & & & $55^{\circ} 07.0^{\prime}$ & $30^{\circ} 10.3^{\prime}$ & $0-100$ \\
\hline 36 & 11 & $8-68$ & & & & & $55^{\circ} 09.5^{\prime}$ & $30^{\circ} 13.4^{\prime}$ & $0-390$ \\
\hline 36 & 12 & $98-16$ & $3-13$ & $3-48$ & & $3-17$ & $55^{\circ} 07.4^{\prime}$ & $30^{\circ} 05.4^{\prime}$ & $0-1140$ \\
\hline 36 & 15 & $5-52$ & & & & & $54^{\circ} 13.5^{\prime}$ & $30^{\circ} 08.8^{\prime}$ & $0-100$ \\
\hline 37 & 4 & $10-175$ & & & & & $53^{\circ} 00.2^{\prime}$ & $29^{\circ} 57.5^{\prime}$ & $300-410$ \\
\hline 37 & 8 & 6-143 & & & & & $52^{\circ} 59.2^{\prime}$ & $29^{\circ} 51.1^{\prime}$ & $195-280$ \\
\hline 37 & 9 & $27-13$ & $2-18$ & $1-?$ & & $8-16$ & $52^{\circ} 58.5^{\prime}$ & $29^{\circ} 47.4^{\prime}$ & $400-1000$ \\
\hline 37 & 11 & 4-109 & & & & & $52^{\circ} 56.9^{\prime}$ & $29^{\circ} 40.7^{\prime}$ & $100-200$ \\
\hline 37 & 12 & $37-111$ & & & & & $52^{\circ} 56.1^{\prime}$ & $29^{\circ} 37.7^{\prime}$ & 45-100 \\
\hline 38 & 1 & 5-168 & & & & & $50^{\circ} 59.8^{\prime}$ & $29^{\circ} 58.2^{\prime}$ & $0-415$ \\
\hline 38 & 6 & 3-74 & & & & & $50^{\circ} 58.9^{\prime}$ & $29^{\circ} 54.8^{\prime}$ & $0-130$ \\
\hline 38 & 8 & $141-25$ & $4-20$ & $12-30$ & & $2-20$ & $50^{\circ} 57.8^{\prime}$ & $29^{\circ} 50.0^{\prime}$ & $0-1005$ \\
\hline 38 & 11 & 6-8 & $7-11$ & $1-11$ & & & $50^{\circ} 53.8^{\prime}$ & $29^{\circ} 43.9^{\prime}$ & $0-520$ \\
\hline 38 & 12 & $8-40$ & & & & & $50^{\circ} 50.4^{\prime}$ & $29^{\circ} 38.6^{\prime}$ & $0-205$ \\
\hline 38 & 14 & $25-37$ & & & & & $50^{\circ} 48.4^{\prime}$ & $29^{\circ} 35.3^{\prime}$ & $0-315$ \\
\hline 39 & 14 & 1-17 & & $3-59$ & & & $47^{\circ} 39.1^{\prime}$ & $30^{\circ} 13.8^{\prime}$ & $500-1020$ \\
\hline 42 & 6 & $4-31$ & & 4-8 & $3-6$ & & $41^{\circ} 44.4^{\prime}$ & $34^{\circ} 20.5^{\prime}$ & $460-870$ \\
\hline 43 & 5 & $2-24$ & $3-14$ & $2-14$ & & & $41^{\circ} 10.3^{\prime}$ & $35^{\circ} 42.4^{\prime}$ & $500-995$ \\
\hline 43 & 7 & $1-23$ & & & & & $41^{\circ} 11.1^{\prime}$ & $35^{\circ} 42.5^{\prime}$ & $0-421$ \\
\hline 45 & 10 & & & $8-21$ & & & $37^{\circ} 08.8^{\prime}$ & $35^{\circ} 01.0^{\prime}$ & $505-1010$ \\
\hline 45 & 11 & $1-?$ & & & $8-13$ & $1-9$ & $37^{\circ} 05.7^{\prime}$ & $35^{\circ} 06.5^{\prime}$ & $385-530$ \\
\hline 45 & 13 & $1-25$ & & & & & $37^{\circ} 03.8^{\prime}$ & $35^{\circ} 09.4^{\prime}$ & $195-380$ \\
\hline
\end{tabular}


TABLE II (continuation)

\begin{tabular}{|c|c|c|c|c|c|c|c|c|c|}
\hline \multirow[t]{2}{*}{ Sta. } & \multirow[t]{2}{*}{ Tr. } & \multirow[t]{2}{*}{ P.p. } & \multirow[t]{2}{*}{ A.v. } & \multirow[t]{2}{*}{ A.w. } & \multirow{2}{*}{$\begin{array}{l}A . p . \\
20 \mathrm{t}\end{array}$} & \multirow{2}{*}{$\begin{array}{l}A . p . \\
24 \mathrm{t}\end{array}$} & \multicolumn{2}{|c|}{ Position } & \multirow{2}{*}{$\begin{array}{l}\text { Depth } \\
\text { (m) }\end{array}$} \\
\hline & & & & & & & North & West & \\
\hline 47 & 3 & $1-31$ & $3-11$ & $1-57$ & & $2-9$ & $35^{\circ} 07.7^{\prime}$ & $31^{\circ} 29.0^{\prime}$ & $425-855$ \\
\hline 47 & 9 & $2-?$ & & & & & $35^{\circ} 06.7^{\prime}$ & $31^{\circ} 16.4^{\prime}$ & $360-520$ \\
\hline 47 & 12 & $2-5$ & $1-16$ & $1-51$ & 4-9 & $1-9$ & $35^{\circ} 07.4^{\prime}$ & $31^{\circ} 07.3^{\prime}$ & $750-1170$ \\
\hline 48 & 8 & $1-7$ & $4-10$ & & $2-9$ & $2-21$ & $34^{\circ} 12.9^{\prime}$ & $31^{\circ} 11.9^{\prime}$ & $500-1150$ \\
\hline 49 & 3 & $2-15$ & & $7-11$ & & & $31^{\circ} 43.1^{\prime}$ & $29^{\circ} 42.6^{\prime}$ & $515-1000$ \\
\hline 49 & 6 & $3-16$ & & & & & $31^{\circ} 44.5^{\prime}$ & $29^{\circ} 35.3^{\prime}$ & 45-107 \\
\hline 49 & 9 & $1-14$ & & & & & $31^{\circ} 47.4^{\prime}$ & $29^{\circ} 30.0^{\prime}$ & $200-325$ \\
\hline 49 & 10 & $2-8$ & & & & & $31^{\circ} 51.2^{\prime}$ & $29^{\circ} 25.2^{\prime}$ & $290-395$ \\
\hline 50 & 2 & & & $2-19$ & $2-13$ & $2-16$ & $30^{\circ} 05.3^{\prime}$ & $29^{\circ} 46.7^{\prime}$ & $730-1200$ \\
\hline 51 & 12 & $1-9$ & $1-13$ & & $2-13$ & $2-32$ & $28^{\circ} 07.0^{\prime}$ & $29^{\circ} 52.8^{\prime}$ & $500-1050$ \\
\hline 52 & 5 & & & $1-21$ & & & $24^{\circ} 57.5^{\prime}$ & $30^{\circ} 01.2^{\prime}$ & $490-1005$ \\
\hline 55 & 4 & & $7-9$ & $2-8$ & $7-7$ & & $27^{\circ} 02.5^{\prime}$ & $20^{\circ} 17.7^{\prime}$ & $570-1000$ \\
\hline \multicolumn{10}{|c|}{ Winter 1982} \\
\hline 62 & 39 & $2-13$ & & $3-19$ & $4-5$ & $2-8$ & $40^{\circ} 56.3^{\prime}$ & $35^{\circ} 33.0^{\prime}$ & 505-980 \\
\hline 62 & 45 & $13-9$ & & & & & $40^{\circ} 56.5^{\prime}$ & $35^{\circ} 40.0^{\prime}$ & $195-305$ \\
\hline 63 & 27 & & $35-8$ & & & & $39^{\circ} 41.9^{\prime}$ & $35^{\circ} 46.2^{\prime}$ & $385-500$ \\
\hline 63 & 28 & & $3-8$ & $6-16$ & & $1-6$ & $39^{\circ} 36.2^{\prime}$ & $35^{\circ} 43.1^{\prime}$ & $505-1000$ \\
\hline 65 & 18 & & $1-11$ & & & & $29^{\circ} 60.0^{\prime}$ & $29^{\circ} 37.5^{\prime}$ & $400-525$ \\
\hline 65 & 20 & & & & & $2-15$ & $29^{\circ} 59.4^{\prime}$ & $29^{\circ} 34.8^{\prime}$ & 490-1010 \\
\hline 66 & 1 & & & & & $4-10$ & $30^{\circ} 00.2^{\prime}$ & $29^{\circ} 29.1^{\prime}$ & $515-995$ \\
\hline
\end{tabular}

taken below $500 \mathrm{~m}$ so that the present findings disagree with previously published lower limits of this species in the water column. The number of specimens collected per trawl fluctuated slightly in the four seasons with a minimum average in autumn of 4 specimens per trawl.

Atolla wyvillei Haeckel, 1880

(Fig. 8)

This widely distributed species occurs over nearly the entire cruise transect, except in summer when it is absent south of $35^{\circ} \mathrm{N}$. In contrast with the preceding species it occurs below 1000 $\mathrm{m}$. It was always collected in trawls below 500 $\mathrm{m}$, except for two trawls from $430-1000 \mathrm{~m}$ and 425-855 $\mathrm{m}$ and an inaccurate open trawl; this species can be considered to live usually below $500 \mathrm{~m}$.

In all seasons the highest abundance is found between $45^{\circ}$ and $50^{\circ} \mathrm{N}$, but numbers are so low that no conclusions can be drawn. The dif- ferences in number, averaging 4.7 specimens per trawl, collected during the different seasons are not significant.

\section{CONCLUSIONS}

In our records Periphylla periphylla is rather a mesopelagic than a typically bathypelagic species; Atolla parva and $A$. wyvillei are bathypelagic species, and $A$. vanhoeffeni is in all probability a deep-mesopelagic species restricted to depth levels of 300-1000 m.

No temperature influences regulating the vertical distribution of coronate medusae have been found.

$A$. parva is a good species. The taxonomic status of the two types with 20 and 24 tentacles at the bell margin cannot be determined on the basis of the present material. Both types occur together in the samples, no essential differences in distribution are found and besides the tentacle number, no other discriminating characters are known. 


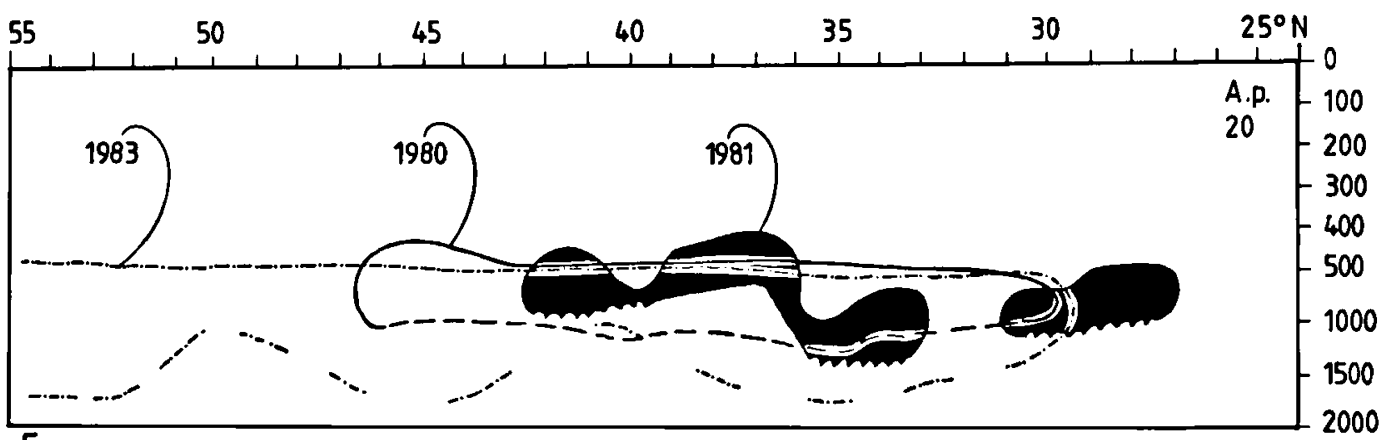

Fig.5

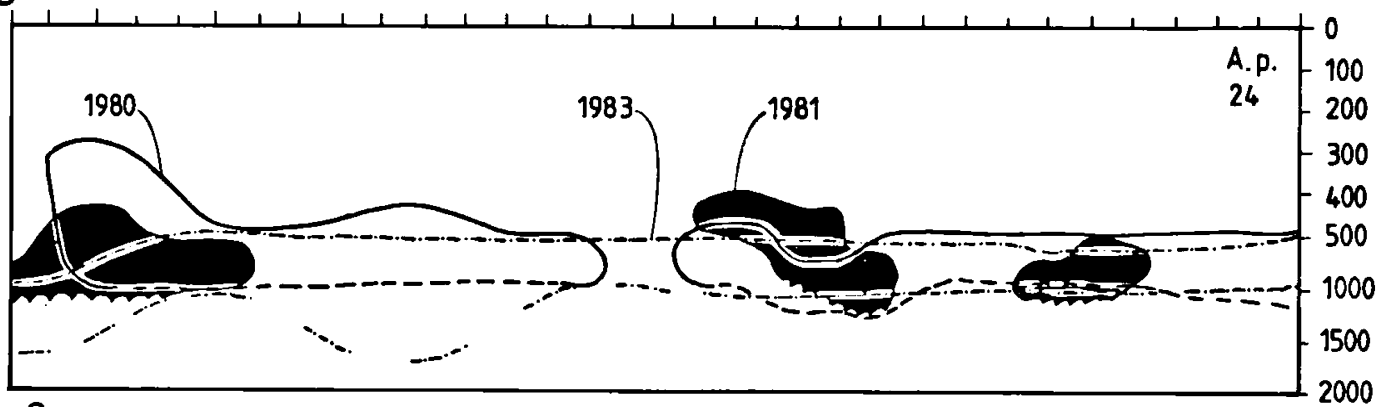

Fig.6

Fig.7
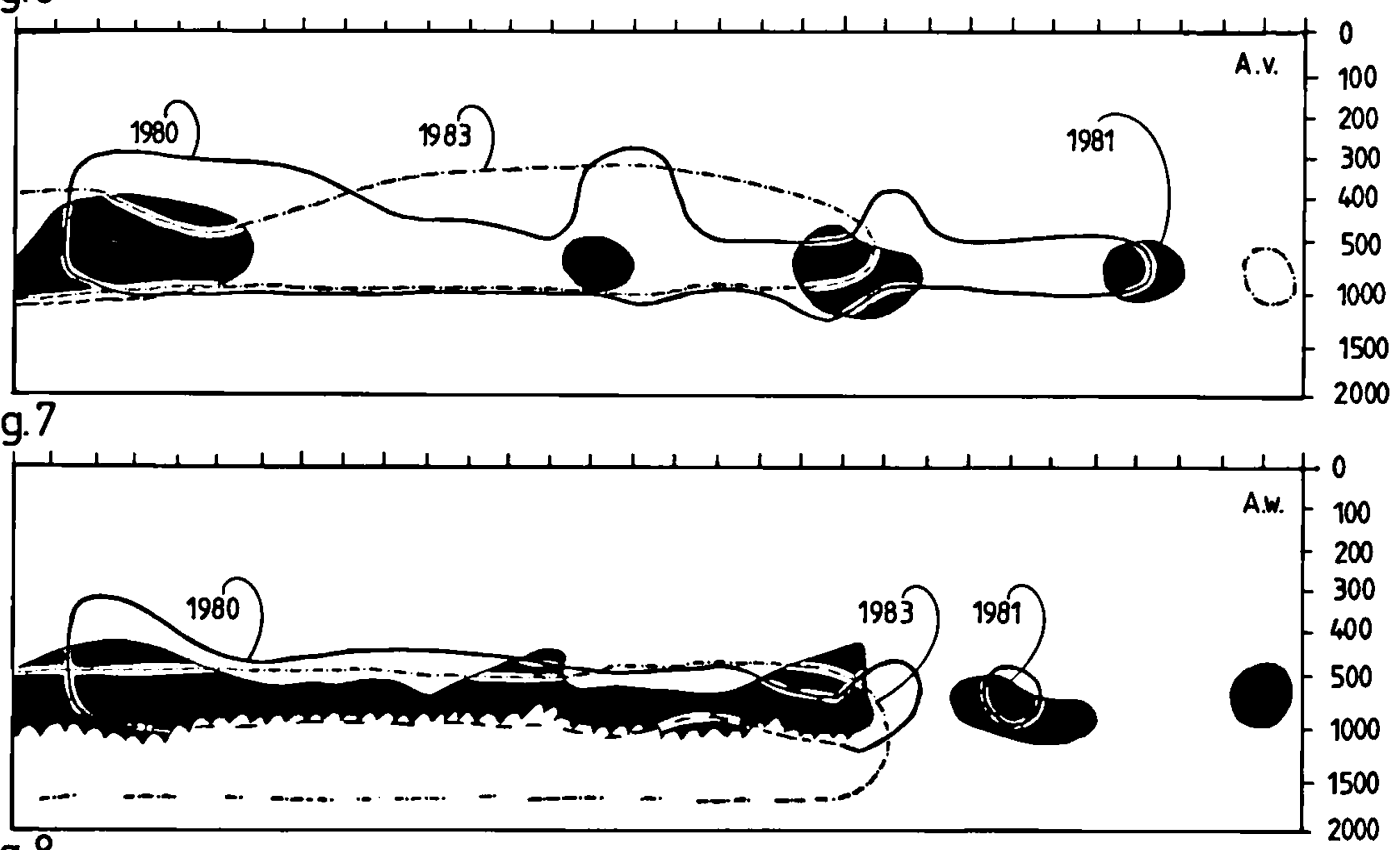

Fig.8

Figs. 5-6. Vertical distribution of Atolla parva along the cruise transect between $55^{\circ} \mathrm{N}$ and $24^{\circ} \mathrm{N}$ for summer 1983 (-.-.-.-), autumn 1981 (black) and spring 1980 (-), given separately for the 20- (fig. 5) and the 24-tentacle form (fig. 6). Depth (m) along the vertical axis.

Fig. 7. Vertical distribution of Atolla vanhoeffeni along the cruise transect between $55^{\circ} \mathrm{N}$ and $24^{\circ} \mathrm{N}$ for summer 1983 (-.-.-..-), autumn 1981 (black) and spring $1980(-)$. Depth (m) along the vertical axis.

Fig. 8. Vertical distribution of Atolla wyvillei along the cruise transect between $55^{\circ} \mathrm{N}$ and $24^{\circ} \mathrm{N}$ for summer 1983 (-.-.-.-), autumn 1981 (black) and spring $1980(-)$. Depth (m) along the vertical axis. 


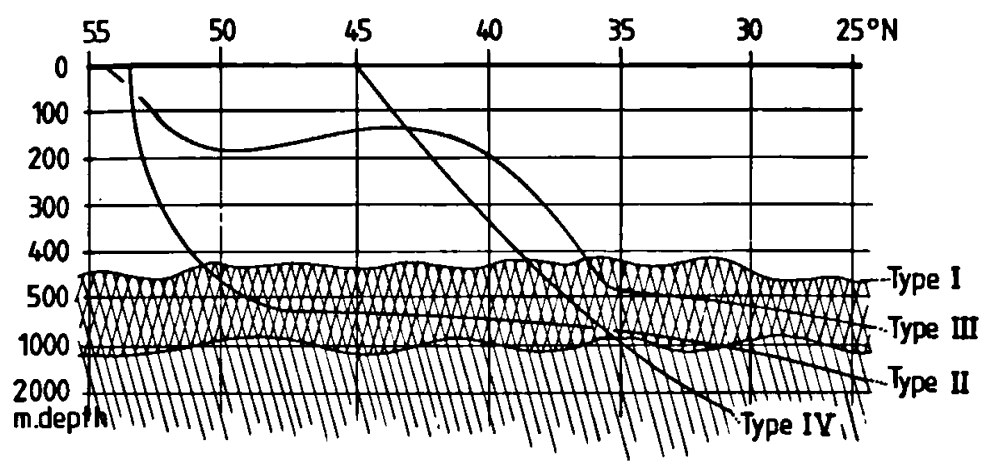

Fig. 9. Diagrammatic representation of the configuration of the four types of upper limits of the bathypelagic realm. The deep-mesopelagic is shown by double hatching (for explanation see text).

The question: "What is the upper level of the bathypelagial?" has more than one answer. At least four types of boundaries can be recognized. In fig. 9 these types are given based on nemerteans, coronates and fishes.

In type I the upper limit of the bathypelagial runs horizontally at a depth of $400-500 \mathrm{~m}$ and seems to be independent of hydrographic conditions. This type is shown by $A$. wyvillei.

Type II shows an upper limit between 500 and $1000 \mathrm{~m}$, probably near $700-800 \mathrm{~m}$ as found for Chaetognatha (cf. Pierrot-Bults, 1982) and crustaceans (cf. Boxshall, 1981), but in colder waters near $55^{\circ} \mathrm{N}$ the upper limit ascends. This distribution seems to be temperaturedependent. Nectonemertes mirabilis shows this pattern as well (cf. Van der Spoel, 1985a). For type II the term deep-mesopelagic should not be used as species like $N$. mirabilis are living also far below $1000 \mathrm{~m}$.

Closely related to this type is type III, where the range comprises shallower depths already north of $35^{\circ} \mathrm{N}$. In some cases this pattern can be temperature-dependent but when, as is shown in fig. 9, the limit stays below $100 \mathrm{~m}$ north of $45^{\circ} \mathrm{N}$ this is not the case. The nontemperature-dependent type III is shown by Periphylla periphylla. This pattern is probably not typically bathypelagic, though Stiasny (1934) described this pattern as such.

Type IV, not found for the present medusae, is essentially different from the other three types, though there is a resemblance to type III.
In pattern IV, the bathypelagic or mesopelagic representatives start to live at shallower depths north of $30^{\circ} \mathrm{N}$ and reach the surface near $45^{\circ} \mathrm{N}$. Sagitta planctonis forma zetesios and the fish Argyropelecus olfersi are the two most carefully studied examples of this pattern (Pierrot-Bults, 1976; Pafort - Van Iersel, 1981).

In fig. 9 the deep-mesopelagic pattern as shown by Atolla vanhoeffeni is indicated by crosshatching. This vertical range seems to coincide partly with the upper layers of the bathypelagial.

Thus it is clear that there is not one single type of upper limit for the bathypelagial, as faunal boundaries are concerned. There is, however, a vertical gradient in which the bathypelagic realm seems to fade out, as is shown in table III. This also explains that there is considerable overlap between the mesopelagial (especially the deep-mesopelagial) and the bathypelagial (cf. table III).

Most bathypelagic representatives in this material are thus found between $1000-1750 \mathrm{~m}$ $\left(1130-1750 \mathrm{~m}\right.$ south of $\left.40^{\circ} \mathrm{N}\right)$, and moderate representation is found between $520-1170 \mathrm{~m}$ $\left(855-1170 \mathrm{~m}\right.$ south of $\left.40^{\circ} \mathrm{N}\right)$, but there are also records though of low diversity between 50 $1200 \mathrm{~m}\left(52-1200 \mathrm{~m}\right.$ south of $\left.40^{\circ} \mathrm{N}\right)$.

The term "subtropical submergence" should not be used to describe the variation in depth distribution as shown e.g. by $P$. periphylla, as no submergence of epipelagic forms is concerned, but a shallower occurrence of deeper living 
TABLE III

Minimum and maximum depth of shallowest point and minimum and maximum depth of deepest point of the trawls with the total number of bathypelagic taxa (coronates and nemerteans) found together in these trawls; depths in parentheses are for trawls taken south of $40^{\circ} \mathrm{N}$ only.

\begin{tabular}{lllll}
\hline $\begin{array}{l}\text { Number of } \\
\text { bathypelagic taxa }\end{array}$ & \multicolumn{2}{l}{$\begin{array}{l}\text { Upper level of net trawls } \\
\text { min. }\end{array}$} & max. & \multicolumn{2}{l}{$\begin{array}{l}\text { Deepest level of net trawls } \\
\text { min. }\end{array}$} & max. \\
\hline 1 & $0(5)$ & $752(752)$ & $50(52)$ & $1005(1005)$ \\
2 & $0(200)$ & $510(515)$ & $98(300)$ & $1090(1090)$ \\
3 & $0(385)$ & $500(730)$ & $50(530)$ & $1200(1200)$ \\
4 & $425(425)$ & $570(570)$ & $885(885)$ & $1150(1150)$ \\
5 & $0(750)$ & 750 & 520 & $1170(1170)$ \\
6 & $0(520)$ & $1000(1000)$ & $1005(1130)$ & $1750(1750)$ \\
7 & $500(770)$ & 1002 & 1000 & $1752(1250)$ \\
\hline
\end{tabular}

taxa. It may be difficult to discriminate between subtropical (near $40^{\circ} \mathrm{N}$ or $\mathrm{S}$ ) submergence and decreasing depth of bathypelagic forms in the same area (type IV), but it strongly differs from shallower occurrence of deep living forms in polar waters (types II \& III).

\section{ACKNOWLEDGEMENTS}

The author is much indebted to C. C. Linnenbank, S. H. Lieuw Kie Song and T. Perquin (Organization for the Advancement of Oceanography in the Netherlands, S.B.N.O.) for their efforts in sorting and identifying part of the material. Commanders and crews of H.M.S. "Tydeman" are kindly acknowledged for their neverfailing help during the collecting cruises.

\section{REFERENCES}

Boxshall, G. A., 1981. Community structure and resource partitioning - the plankton. In: P. L. Forey ed., The evolving biosphere: 143-156 (British Museum (Natural History) \& Cambridge University Press, London/Cambridge/New York/Melbourne).

Casanova, J. P., 1977. La faune pélagique profonde (zooplancton et necton) de la Province atlantoméditerranéenne. Aspects taxonomiques, biologiques et zoogéographiques: 1-455 (Thèse Doct. État Univ. Provence, Marseille).

——, 1981. Nouvelles formulations des règles écologiques connues sous le nom de règle de Bergmann et loi de Jordan. J. Plankton Res., 3 (4): 509-529.

EkMAN, S., 1953. Zoogeography of the sea: i-xiv, 1-417 (Sidgwick \& Jackson, London).

Heyman, R. P., 1981. Narcotisation, fixation and preser- vation experiments with marine zooplankton. Verslagen en technische Gegevens, Inst. taxon. Zoöl. (Zoöl. Mus.) Univ. Amsterdam, 28: 1-36.

KRAMP, P. L., 1913. Medusae collected by the "Tjalfe" Expedition. Vidensk. Meddr. dansk naturh. Foren., 65: 257-286.

- 1 1947. Medusae. Part III.Trachylina and Scyphozoa with zoogeographical remarks on all the medusae of the northern Atlantic. Danish Ingolf Exped., 5 (14): $1-66$.

Marshall, N. B., 1979. Developments in deep-sea biology: i-x, 1-566 (Blandford Press, Dorset).

Mauchline, J. \& P. F. Harvey, 1983. The Scyphomedusae of the Rockall Trough, north eastern Atlantic Ocean. J. Plankton Res., 5 (6): $881-890$.

Pafort - VAN IERSEL, T., 1981. The Sternoptychidae (Pisces, Stomiatoidei) of the Amsterdam Mid North Atlantic Plankton Expedition 1980, with a note on specimens intermediate between Argyropelecus aculeatus Valenciennes, 1849 and A. olfersi (Cuvier, 1829). Beaufortia, 31 (4): 97-106.

Pierrot-Bults, A. C., 1976. Zoogeographic patterns in chaetognaths and some other planktonic organisms. Bull. zool. Mus. Univ. Amsterdam, 5 (8): 59-72.

- 1982 . Vertical distribution of Chaetognatha in the Central Northwest Atlantic near Bermuda. Biol. Oceanogr., 2 (1): 31-61.

Russell, F. S., 1959. Some observations on the Scyphomedusa Atolla. J. mar. biol. Ass. U.K., 38 (1): 33-40.

-, 1970 . The medusae of the British Isles, II: i-xi, 1284 (Cambridge Univ. Press, Cambridge).

SPOEL, S. VAN DER, 1981. List of discrete depth samples and open net hauls of the Amsterdam Mid North Atlantic Plankton Expedition 1980 (Project 101A). Bull. zool. Mus. Univ. Amsterdam, 8 (1): 1-10.

-, 1985 a. Pelagic nemerteans of the Amsterdam Mid 
North Atlantic Plankton Expeditions (AMNAPE), 1980-1983. Beaufortia, 35 (2): 15-24.

- , 1985b. List of discrete depth samples and open net hauls of the Amsterdam Mid North Atlantic Plankton Expeditions 1982 and 1983 (Project 101A). Bull. zool. Mus. Univ. Amsterdam, 10 (17): 129-152.

Spoel, S. van DER \& R. P. Heyman, 1983. A comparative atlas of zooplankton. Biological patterns in the oceans: 1-186 (Bunge, Utrecht).
Spoel, S. van der \& A.G. H. A. Meerding, 1983. List of discrete depth samples and open net hauls of the Amsterdam Mid North Atlantic Plankton Expedition 1981 (Project 101A). Bull. zool. Mus. Univ. Amsterdam, 9 (9): 77-91.

Stiasny, G., 1934. Scyphomedusae. Discovery Rep., 8: 329-396.

Vinogradov, M. E., 1970. Vertical distribution of the oceanic zooplankton: 1-339 (Israel Program for scientific Translations, Jerusalem). 